\title{
Rare finding in a patient with Mycosis fungoides - sensorimotor axonal polyneuropathy
}

\author{
Oana Obrisca' ${ }^{1}$, Alexandru Bardas², Oana Morari', Ayghiul Elmi-Mujdaba', \\ Bogdan 0. Popescu ${ }^{1,3,4}$, Mariana Mihaila ${ }^{5}$ \\ ${ }^{1}$ Department of Neurology, Colentina Clinical Hospital, Bucharest, Romania \\ ${ }^{2}$ Department of Hematology, Fundeni Clinical Institute, Bucharest, Romania \\ ${ }^{3}$ Department of Neurology, "Carol Davila" University of Medicine and Pharmacy, Bucharest, Romania \\ ${ }^{4}$ Department of Molecular Medicine, "Victor Babes" National Institute of Pathology, \\ Bucharest, Romania \\ ${ }^{5}$ Department of Internal Medicine, Fundeni Clinical Institute, Bucharest, Romania
}

\begin{abstract}
We report the case of a 62-year old patient who presented a rare association of Mycosis fungoides, a subtype of cutaneous T-cell lymphoma and sensorimotor axonal neuropathy. Usually, leptomeningeal dissemination and central nervous system involvement are much more frequently encountered in cases of Mycosis fungoides with extracutaneous spread and only few case reports describe the involvement of peripheral nerves. The challenge in our case was to correlate a possible etiopathogenic factor with the polyneuropathy. Although, it was most probably a paraneoplastic manifestation, the associated neuropathy could have had other possible pathogenic mechanisms.
\end{abstract}

Keywords: cutaneous lymphoma, sensorimotor axonal neuropathy, Mycosis fungoides

\section{INTRODUCTION}

Neurological disorders secondary to neoplasia are highly variable, since they can involve both the central and the peripheral nervous system. Paraneoplastic neuropathies are of great importance, since their symptomatology can precede the manifestations of a malignant process, guiding the physician to an early diagnosis of neoplasia. (1) Sometimes, it is difficult to point out the exact etiology of a neuropathy in the context of a neoplasm, because several mechanisms responsible for nerve destruction can interfere, such as neuroimmunological processes, neurotoxicity of the chemotherapy, malnutrition and thiamine deficiency or nerve metastasis.

Among hematologic malignancies, cutaneous T-cell lymphomas (CTCL) are rarely associated with neurological complications, but when extracu- taneous spread takes place, leptomeningeal dissemination and CNS involvement are more frequently encountered than paraneoplastic neuropathy. (2)(3) Mycosis fungoides (MF) and Sezary syndrome (SS) account for almost two thirds of CTCL and the main difference between them is the leukemic involvement specific for SS, with dissemination of Sezary cells in blood, lymph nodes and bone marrow. (4) Few risk factors associated with MF/SS have been identified, such as HTLV 1 infection and hydrochlorothiazide (drug-induced pseudolymphoma). (5)

\section{CASE PRESENTATION}

A 62-year-old man presented to our clinic with erythroderma, peripheral lymphadenopathies, sig- 
nificant weight loss $(10 \mathrm{~kg})$ over the last 6 months and walking difficulties, accompanied by distal paresthesia. The patient noticed skin changes 8 months ago, when keratotic plaques appeared on both his arms. The desquamative plaques progressed rapidly to generalized erythroderma during the next 4 months. There was a history of a preceding skin lesion, a desquamative patch on his left elbow, which disappeared spontaneously 2 years ago. The patient was a heavy smoker (40 packyears), consumed alcoholic drinks occasionally and had professional exposure to pesticides. The past medical history included chronic obliterative arteriopathy and chronic obstructive pulmonary disease.

Physical examination revealed a relatively good general status, normal body temperature, generalized erythroderma, intensely desquamative and pruritogenic skin (Fig. 1), dystrophic nails, palpable, firm axillary and inguinal lymph nodes, blood pressure $130 / 80 \mathrm{mmHg}$ and pulse $90 / \mathrm{min}$. The auscultation revealed regular heartbeats and normal breath sounds, and the abdominal palpation showed no signs of organomegaly. The neurological examination consisted of a walking perimeter under 500 $\mathrm{m}$, weakness in lower limbs, bilateral absence of patellar reflexes, mild atrophy of the calf muscles, paresthesia in lower limbs, with normal sensory function, no signs of dysmetria and normal cranial nerve testing.

Laboratory testing revealed a normal WBC count of $6,600 / \mu \mathrm{L}$, with a slightly eosinophilia, hemoglobin level of $12.9 \mathrm{~g} / \mathrm{dL}$, normal liver function tests and renal function, negative viral hepatitis markers, negative HIV test, negative HTLV-1 determination and serum B12 level at the lower range of normal value $(190 \mathrm{pg} / \mathrm{ml})$. The lipid panel displayed serum cholesterol and triglycerides in normal range. There were signs of mild inflammation (C reactive protein $20.2 \mathrm{mg} / \mathrm{L}$, ESR $50 \mathrm{~mm} / \mathrm{h}$ ), with normal procalcitonin level. The NSE [neuron-specific enolase) was elevated $(68.43 \mathrm{ng} / \mathrm{ml})]$.

An extended CT scan displayed mediastinal, bilateral axillary and inguinal adenopathies (13-30 $\mathrm{mm}$ ), thickening of the bronchi walls, associated with bilateral small condensation areas, 2 pulmonary nodules (4 $\mathrm{mm}$ and $9 \mathrm{~mm}$ in diameter) in the left lung parenchyma, a normal spleen of $92 / 43 \mathrm{~mm}$ and a steatotic liver. After 6 months, a second thoracic $\mathrm{CT}$ was performed and the two pulmonary nodules did not progress in dimensions. The patient underwent upper and lower gastrointestinal endoscopy, which revealed sigmoid diverticula and external haemorrhoids, with no signs of neoplasia.

The nerve conduction studies displayed a pattern suggestive of mixed asymmetrical axonal polineuropathy, present in both upper and lower limbs, but with a greater involvement in the lower limbs (right $>$ left). (Table 1)

The following investigations consisted of skin and lymph node biopsies. The skin biopsy revealed irregular psoriasiform acanthosis, spongiosis areas with lymphocytic exocytosis and a dense lymphocytic infiltrate in the reticular dermis, with small, atypical lymphocytes. The histologic features resembled chronic dermatitis, but also raised the sus-

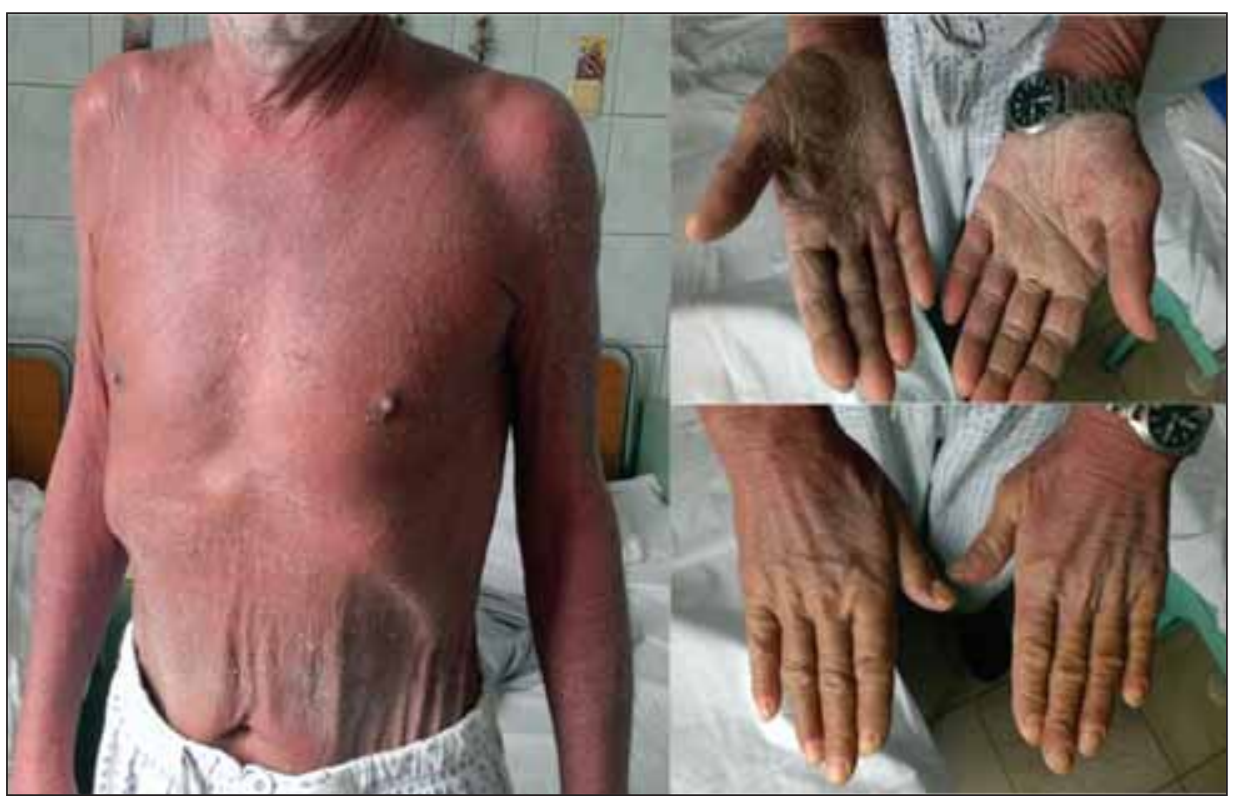

FIGURE 1. Generalized erythroderma, with desquamative lesions and dystrophic nails 
TABLE 1. Summary EMG data

\begin{tabular}{|l|c|c|c|c|}
\hline Stimulation site & Latency (ms) & $\begin{array}{c}\text { Ampl } \\
(\mathbf{m V})\end{array}$ & $\begin{array}{c}\text { Duratio } \mathbf{n} \\
(\mathbf{m s})\end{array}$ & $\begin{array}{c}\text { Velocity } \\
\text { (m/s) }\end{array}$ \\
\hline 1. R, Abductor digiyi minimi, Ulnaris & & & & \\
\hline wrist & 3.3 & 7.2 & 6.28 & \\
\hline elbow & 7.2 & 6.9 & 5.6 & 67.7 \\
\hline 2. R, Abductor pollicis brevis, Medianus & & & & \\
\hline wrist & 3.6 & 6.1 & 6.72 & \\
\hline elbow & 8.1 & 6.4 & 7.04 & 57.5 \\
\hline 3. R, Tibialis anterior, Peroneus & & & & \\
\hline head of fibula & 4.3 & 0.8 & 9.96 & \\
\hline popliteal fossa & 5.5 & 0.9 & 10.1 & 83.3 \\
\hline 4. L, Tibialis anterior, Peroneus & & & & \\
\hline head of fibula & 4.4 & 1.8 & 14.1 & \\
\hline popliteal fossa & 5.4 & 1.9 & 14 & 109 \\
\hline 5. R, Abductor hallucis, Tibialis & & & & \\
\hline medial malleolus & 4.9 & 1.9 & 5.04 & \\
\hline popliteal fossa & 13.2 & 1.5 & 6.44 & 48.1 \\
\hline 6. L, Abductor hallucis, Tibialis & & & & \\
\hline medial malleolus & 5.6 & 3.3 & 3.72 & \\
\hline popliteal fossa & 14.9 & 3 & 3.96 & 42.7 \\
\hline Sensory CV: & 2.4 & 13.9 & 1.6 & 50.4 \\
\hline 1. R, Ulnaris - wrist & 2.4 & 5.2 & 1.5 & 58.3 \\
\hline 2. R, Medianus - wrist & 56.9 & 4.4 & 1.2 & 25.8 \\
\hline 3. R, Peroneus Superficialis - middle third of leg & 56.6 & & & 42.3 \\
\hline 4. R, Suralis & & & & \\
\hline F-wave parameter: & & & & \\
\hline R, Abductor pollicis brevis, Medianus & & & \\
\hline R, Abductor hallucis, Tibialis & & & \\
\hline L, Abductor hallucis, Tibialis & & & \\
\hline & & & & \\
\hline
\end{tabular}

picion of cutaneous lymphoma. After immunohistochemical staining, the majority of the lymphocytes were $\mathrm{CD} 3+, \mathrm{CD} 2+, \mathrm{CD} 5+, \mathrm{CD} 7+$ and the $\mathrm{CD} 4 /$ CD8 ratio was 6-7:1. Monoclonal rearrangement of T-cell receptor (TCR) gamma genes was present in a low grade at immunogenotyping exam. Lymph node biopsy showed chronic reactive dermatopathic changes, without atypical lymphocytes or neoplastic cells. In addition, bone marrow analysis displayed no signs of abnormal cell proliferation and blood flow cytometry showed no abnormal lymphocytic populations.

\section{Discussion}

First task in evaluating our patient was to differentiate between a paraneoplastic erythroderma, secondary to lung carcinoma or a primary cutaneous neoplasia. The pulmonary nodules, in the context of heavy smoking and exposure to pesticides, accompanied by the elevated level of NSE (6) raised the suspicion of small-cell lung carcinoma. The patient refused the bronchoscopic alveolar lavage in order to exclude pulmonary neoplasia. However, taking into consideration that the two nodules re- mained stationary between the two thoracic CT scans, such an indolent progression would have been improbable for small-cell lung carcinoma.

On the other hand, there were important clinical and paraclinical arguments suggestive of primary cutaneous neoplasia. The skin changes described at light microscopy were not specific for cutaneous lymphoma, but the immunohistochemical staining showed an abnormal CD4/CD8 ratio of 6-7:1 (a CD4/CD8 ratio of 10:1 - ISCL criteria for Sezary syndrome) (5). That finding, corroborated with the monoclonal rearrangement of TCR gamma genes was highly suggestive for Mycosis fungoides (MF). In several cases, the biopsy cannot offer a definitive diagnosis of MF and instead, offers information "suggestive of" MF. In these situations, the diagnosis can be challenging and is established mostly on clinicopathological correlations. (5) Nevertheless, the median time from symptom onset to diagnosis is 3 to 4 years, so the course of our patient's lesions matches the indolent evolution of this type of CTCL. (5) The presence of generalized erythroderma without secondary dissemination in lymph 
nodes or other sites, places our patient in stage III MF. (4)

The next step in our diagnostic assessment was to correlate the neuropathy with a possible etiological factor. From previous case reports, the involvement of the peripheral nerves in MF cases seems to be rare, because more frequently a leptomeningeal dissemination is described, along with CNS affections. $(7,8)$ Keeping in mind this aspect, we first tried to eliminate other possible causes of neuropathy in our patient. There was no history of diabetes, of heavy drinking or administration of toxic medications. In addition, the patient did not undergo chemotherapy at the moment of diagnosis. The serum B12 level was at the lower range of normal, but there were no signs of macrocytosis, the homocystinuria and the seric methylmalonic acid were within normal range.

Instead, there were certain clinical clues that indicated a correlation between the extension of the erythroderma and the onset of the peripheral neuropathy. The walking difficulties and leg weakness progressed rapidly along with the generalized erythroderma, resembling a subacute evolution. The expansion of the cutaneous lymphoma was responsible of peripheral nerve distruction, but unfortunately, it was impossible for us to point out whether a neuroimmunological process or a direct neoplastic invasion of the nerves was the pathogenic mechanism.

Neurological complications are rarely encountered in CTCL and they mainly consist of meningeal infiltration in patients with advanced MF/SS, with a poor prognosis. In a retrospective study of 187 patients with CTCL, 3\% of them had a neoplastic neurological disorder and at least half of the neurological complications were due to direct involvement of the nervous system. (3) That makes paraneoplastic polyneuropathy a rare finding in MF cases. Bezier et al (8) described the first case of neurolymphomatosis proven by histologic examination in a 73-year old patient with Sezary syndrome and acute axonal poliradiculoneuropathy. At nerve biopsy, the epineural artery and endoneurium were infiltrated with Sezary cells. The particularity of our case is that the patient's cutaneous lymphoma was limited to the skin, without extracutaneous involvement. Therefore, a nerve biopsy would have been the key investigation in determining if atypical T lymphocytes invaded the vasa nervorum even in a more indolent stage of MF, in a similar way as it was previously described in advanced forms. Unfortunately, the patient refused to undergo nerve biopsy.

Since we did not have the means to detect the serum onconeural antibodies (anti-Hu, anti-CV2), we have no data regarding the neuroimmunological pathway. Paraneoplastic sensorimotor neuropathy associated with onconeural antibodies can be present in lung cancer, Hodgkin lymphoma, nonHodgkin lymphoma with monoclonal proteins and myeloma, but it usually has a demyelinating pattern. In contrast, axonal sensorimotor neuropathy, such as the one described in our case, is also a common finding in patients with malignancies, but in most cases, the paraneoplastic antibodies are absent. Therefore, it is hard to establish with certainty if an axonal mixed neuropathy in a cancerous patient is truly a paraneoplastic manifestation or is more related to toxic, metabolic or nutritional causes. (1)

However, the progression of the neuropathy was somehow atypical, because, from a certain point, when the erythrodermic transformation was complete, the symptomatology stopped evolving. Theoretically, this could be the expression of a pathogenic process, which was intensely active during the cutaneous spread of neoplastic epidermotropic $\mathrm{T}$ lymphocytes and in time reduced its intensity, therefore limiting the damage of the nerve tissue. At the moment of diagnosis, the nerve damage was already installed and did not progress any further.

Regarding the therapeutic management, our first approach consisted of skin-directed therapies, such as PUVA (psoralen plus ultraviolet A radiation) in order to control skin lesions, avoiding the toxicity of chemotherapeutic agents. (9) The patient responded well to the treatment, because the skin lesions started to regress and the discomfort was ameliorated. Also, paresthesia and walking difficulties improved, so we did not have to recur to more aggressive immunosuppressive therapies.

In conclusion, the case presented above illustrates how challenging it is for a clinician to assess the aetiological factors responsible for nerve distruction in a patient with a malignant condition. Also, the association between Mycosis fungoides in a stage limited to the skin and sensorimotor axonal neuropathy provides further evidence that paraneoplastic affections of the peripheral nervous system can be present in CTCL. 


\section{REFERENCES}

1. Muppidi S. and Vernino S. Paraneoplastic Neuropathies, pp. 1359-1372, 2004.

2. Karadağ Y.S., Gülünay A., Oztekin N., F. Ak, and Kılıçkap S. Peripheral neuropathy in Sézary syndrome: coincidence or a part of the syndrome?, Turkish J. Haematol. Off. J. Turkish Soc. Haematol., vol. 30, no. 4, pp. 420-1, Dec. 2013.

3. Kaufman D.K., Habermann T.M., Kurtin P.J., O'Neill B.P. Neurological complications of peripheral and cutaneous T-cell lymphomas, Ann Neurol, vol. 36, pp. 625-629, 1994.

4. Olsen E., Vonderheid E., Pimpinelli N. Revisions to the staging and classification of mycosis fungoides and Sezary syndrome: a proposal of the International Society for Cutaneous Lymphomas (Blood, vol. 110, no. 6, pp. 1713-1723, 2007.

5. Wilcox R.A. Cutaneous T-cell lymphoma: 2016 update on diagnosis, risk-stratification, and management, Am. J. Hematol., vol. 91, no. 1, pp. 151-65, Jan. 2016.
6. Wang H., Zhang X., Liu X., Liu K., Li Y., Xu H. Diagnostic value of bronchoalveolar lavage fluid and serum tumor markers for lung cancer, J. Cancer Res. Ther., vol. 12, no. 1, p. 355, 2016.

7. Donovan R.C., Freeman C. Case Report Brainstem Involvement With Mycosis Fungoides : An Unusual Central Nervous System Complication, no. April, pp. 533-534, 2002.

8. Bezier M., Delaby P., Laroche L., Bernard P., Grange F. Neurolymphomatosis Associated With Sezary Syndrome, vol. 145, no. 3, pp. 294-296, 2009.

9. Prince H.M., Whittaker S., Hoppe R.T., How I treat mycosis fungoides and Sézary syndrome, Blood, vol. 114, no. 20, pp. 4337-53, Nov. 2009. 